\title{
Communication \\ Cortisol Reactivity to a Digital Version of Trier Social Stress Test and Eating Behavior in Non-Overweight and Overweight Adolescents: A Pilot Study
}

\author{
Ioan Gotca ${ }^{1,2}$, Andrada Druica ${ }^{1,2, *}$, Daniel V. Timofte ${ }^{3,4, *}$, Cristina Preda ${ }^{5}$, Dana-Teodora Anton-Paduraru ${ }^{6} \mathbb{D}$, \\ Cristina Mihaela Ghiciuc ${ }^{7}\left({ }^{(}\right.$, Maria Christina Ungureanu ${ }^{5}$, Letitia Leustean ${ }^{5}$ and Veronica Mocanu $1,2, *(1)$
}

1 Department of Morpho-Functional Sciences II (Pathophysiology), “Grigore T. Popa” University of Medicine and Pharmacy, 16, Universitatii Street, 700115 Iasi, Romania; ioan.gotca@umfiasi.ro

2 Center for Obesity BioBehavioral Experimental Research, 16, Universitatii Street, 700115 Iasi, Romania

3 Department of Surgery, "Grigore T. Popa" University of Medicine and Pharmacy, 16, Universitatii Street, 700115 Iasi, Romania

4 Bariatric Surgery Center, Clinical Emergency County Hospital “St. Spiridon", 1, Independentei Street, 700111 Iasi, Romania

5 Department of Medical Sciences II (Endocrinology), “Grigore T. Popa” University of Medicine and Pharmacy, 16, Universitatii Street, 700115 Iasi, Romania; cristina.preda@umfiasi.ro (C.P.);

maria.ungureanu@umfiasi.ro (M.C.U.); letitia.leustean@umfiasi.ro (L.L.)

check for

updates

Citation: Gotca, I.; Druica, A.;

Timofte, D.V.; Preda, C.;

Anton-Paduraru, D.-T.; Ghiciuc, C.M.;

Ungureanu, M.C.; Leustean, L.;

Mocanu, V. Cortisol Reactivity to a

Digital Version of Trier Social Stress

Test and Eating Behavior in

Non-Overweight and Overweight

Adolescents: A Pilot Study. Appl. Sci.

2021, 11, 9683. https://doi.org/

10.3390/app11209683

Academic Editors: Francisco Arrebola,

Alessandra Durazzo and Wojciech

Kolanowski

Received: 31 August 2021

Accepted: 12 October 2021

Published: 17 October 2021

Publisher's Note: MDPI stays neutral with regard to jurisdictional claims in published maps and institutional affiliations.

Copyright: $\odot 2021$ by the authors. Licensee MDPI, Basel, Switzerland. This article is an open access article distributed under the terms and conditions of the Creative Commons Attribution (CC BY) license (https:// creativecommons.org/licenses/by/ $4.0 /)$.
6 Department of Mother and Child Medicine (Pediatrics), University of Medicine and Pharmacy, 16, Universitatii Street, 700115 Iasi, Romania; dana.anton@umfiasi.ro

7 Department of Morpho-Functional Sciences II (Pharmacology, Clinical Pharmacology and Algesiology), “Grigore T. Popa" University of Medicine and Pharmacy, 16, Universitatii Street, 700115 Iasi, Romania; cristina.ghiciuc@umfiasi.ro

* Correspondence: andrada-t-murgoci@d.umfiasi.ro (A.D.); daniel.timofte@umfiasi.ro (D.V.T.); veronica.mocanu@umfiasi.ro (V.M.)

Abstract: (1) Background: In the present pilot study, we examined the response of cortisol to a digital version of the Trier Social Stress Test (TSST) and corresponding eating preferences in non-overweight and overweight adolescents. (2) Methods: The experimental study group included 35 adolescents aged $15.7 \pm 0.5$ years (16 boys and 19 girls). The participants were split into two groups: nonoverweight $(\mathrm{N}=24)$ and overweight $(\mathrm{N}=11)$, according to the Body Mass Index (BMI). We induced acute stress in all participants, using a digital version of the Trier Social Stress Test (TSST), with three different digital tasks. We measured salivary cortisol before the test (T0), during the stress induction by digital tasks (T5, T10, T15), and 25 min after the stress test (T40). The Health Behavior in School-Aged Children Questionnaire (HBSC) was administered to assess the influence of stress on eating behavior: the consumption of fruit, vegetables, sweets, and soft drinks and the frequency of snacks. (3) Results: Among the entire group of adolescents, we observed a significant difference between the sexes in terms of cortisol response, girls being predominantly hyperreactive (13 girls compared to 5 boys, $p=0.03$ ). In overweight adolescents, the cortisol reactivity was lower in boys and higher in girls $(p=0.05)$. The overweight hyperreactive adolescents, as compared to the hyporeactive ones, had a higher frequency of daily consumption of sweets $(25 \%$ vs. $0 \%)$ and soft drink ( $25 \%$ vs. 0$)$. (4) Conclusions: The individual patterns of cortisol reactivity to laboratory-induced stress could be associated with an increased risk of unhealthy eating behavior in adolescents.

Keywords: Trier social stress test; digital technique; adolescent; salivary cortisol; eating behavior

\section{Introduction}

There is a positive association between stress and obesity [1]. Obesity has been linked to disturbances of the hypothalamic-pituitary-adrenal (HPA) axis and an abnormal cortisol output [2]. Cortisol is the glucocorticoid end product of the HPA system. An essential aspect of the body's interconnected set of physiological systems for responding 
to challenges, the HPA axis is especially sensitive to stressful situations, especially those involving novelty, uncontrollability, or social threat [3].

Stressful events have been associated with both increased and decreased food intake $[4,5]$, and the biological mechanism involves interactions between glucocorticoids, appetite-related hormones (e.g., ghrelin, leptin, insulin), and the brain's dopaminergic reward system [6]. Thereby, coping strategies to manage stress can lead to, on the one hand, increased food intake with a lot of sugary/fatty food or, on the other hand, avoidance of food in the context of a 'fight-or-flight reaction' [5].

Stress-induced eating and cortisol reactivity to an acute stressor might be related to eating behavior [7]. Depending on their cortisol reactivity to a stressor, subjects may be divided into high and low reactors [5]. Laboratory studies of the link between stress reactivity in obese individuals have yielded mixed results. Observations include heightened [4], blunted [6], or no differences [8,9] in HPA axis stress responses in obese groups compared with normal-weight individuals. These divergent results might be explained by methodological differences, especially the nature of the stressor and the lack of a control group.

Previous studies suggest that social stress is a potent determinant of unhealthy food choices in adolescents with excess weight $[10,11]$. This phenomenon could be explained by the harmful impact of social stress on eating-related behaviors in adolescents [10]. Higher cortisol reactivity [11,12], blunted cortisol response [13], or no differences in cortisol response [14] to the Trier Social Stress Test (TSST) were found in adolescents with excess weight as compared to the normal-weight group.

Fewer studies assessed the relationship between cortisol reactivity and eating behavior in adolescents. We aimed to find the patterns of eating behavior in non-overweight and overweight adolescents with hypo or hyper-reactivity of cortisol to experimentally induced acute stress.

\section{Design and Methods}

\subsection{Study Population}

Thirty-nine adolescents aged between 15 and 17 years old were enrolled in this pilot study (18 boys and 21 girls). Participants were recruited from a high school located in Iași (Romania). The inclusion criteria for participants were defined as follows: (i) age range between 15 and 17 years old; (ii) for girls, to be in the luteal phase of the menstrual cycle at the time of testing and not to use hormonal contraceptives; (iii) absence of history or current evidence of physical or psychiatric disorders. Four participants provided invalid cortisol samples, and therefore, the final study sample comprised 35 adolescents (16 boys and 19 girls). Data were gathered in October 2019.

The 35 participants were split into two groups: non-overweight $(\mathrm{N}=24)$ and overweight $(\mathrm{N}=11)$, according to the Body Mass Index (BMI). The classification of the two groups was conducted in alignment with World Health Organization (WHO) guidelines. Based on the WHO Reference 2007 [15], in children aged 5-19 years, the participants were considered non-overweight (BMI-for-age value $<+1 \mathrm{SD}$ ) or overweight (BMI-for-age value $\geq+1 \mathrm{SD})$.

\subsection{Study Design}

The study procedure started at 09:00 $\mathrm{h}$, and the adolescents were exposed to a novel digital version of the Trier Social Stress Test (TSST). The TSST was conducted by the same examiner (I.G.). Salivary cortisol concentrations were determined at 5 consecutive time points: 1 baseline time point before the TSST (T0), 3 time points during the test (T5, T10, and T15), and $25 \mathrm{~min}$ following test completion (T40). The perceived stress assessment, as described below, was performed just before the initiation of the TSST and immediately following it. The Health Behavior in School-Aged Children Questionnaire (HBSC) [16] was applied to identify age, weight, sociodemographic variables, and lifestyle factors, including nutrition practices, physical activity and sedentary behaviors, perceived well-being, and health complaints. 


\subsection{Trier Social Stress Test (TSST)}

We induced acute stress in all participants using a digital version of the TSST. The studied adolescents were students enrolled in the same high school. An overview of the study was provided before the test during the classes by the principal investigator, and individuals interested in participating were given informed consent to be signed. On the day of the experiment, the participants were informed about the digital tasks they were to perform during the stress test. The experiment was conducted in the high school between 8:00 and 13:00. They were tested individually in a familiar room (a schoolroom) for a total duration of $60 \mathrm{~min}$. Girls were tested in the luteal phase to standardize the influence of the menstrual cycle [9].

\subsubsection{Pre-Experimental Stage (10 $\mathrm{min})$}

Pre-test Perceived Stress Scale (Pre-test PSS). The level of psychological stress was assessed by using the Perceived Stress Scale (PSS) designed by Cohen [17] and adapted for children and adolescents. The PSS consists of 10 items. To evaluate the pre-test perception of test difficulty, we included seven questions (e.g., "Do you happen to face situations where you feel maximum pressure?", "Do the emotions you feel influence you negatively in many situations?", "Do you usually have a state of discomfort before having a problem?"). The first saliva sample was collected before the experimental stress test.

\subsubsection{Experimental Stage}

The stress induction stage started when the participants entered a room where a committee of three persons (female and male members) dressed in white lab coats were waiting. The room was equipped with an audio-video recording system. Each digital test had a duration of $5 \mathrm{~min}$. The saliva collections were performed at the end of each digital test.

The first digital test $(5 \mathrm{~min})$. A digital spatial representation test was used to induce psychological stress. The test was designed in the shape of a narrow path with a curved line to be solved in a given time of $5 \mathrm{~min}$, by which average psychological stress could be induced. The test involved following a line marked on the screen of a tablet without deviating from the set path. If there was a small deviation, an unpleasant sound was produced and the participants were obliged to start the test from the beginning.

The second digital test $(5 \mathrm{~min})$. The test induced stress by asking the participants to make arithmetic subtraction (sequentially subtracting 13 from 1022) in front of the committee and to communicate the result aloud. If the result of the calculation was incorrect, the electronic device verbally informed the participants that they were wrong and that they had to resume the arithmetic subtraction calculations from the standard number.

The third digital test $(5 \mathrm{~min})$. This test induced stress by asking the participants to find solutions to escape from a room puzzle (a large rectangle divided into five "rooms").The objective of the puzzle was to cross each "wall" of the diagram only once with a continuous line. Subjects could start where they wanted and had an unlimited number of tests available.

\subsubsection{The Recovery Stage ( $25 \mathrm{~min})$}

Post-test Perceived Stress Scale (Post-test PSS). In the post-experimental phase, the participants completed the 10-item PSS, which was adapted to measure the effects of the stressful events of the current day. To evaluate the post-test perception of test difficulty, we included seven items (e.g., "The tests were too difficult, in my opinion", "I am sure I have successfully solved the tests", "I would be happy if I could retake the tests when I will be in a better mood"). The range of total scores for the PSS (17 items) was from 0 to 68 . The higher the score, the greater the severity of perceived stress. In our study, the cutoff value between the stressed and the unstressed students, based on quartiles, was 31 . 
Health Behavior in School-Aged Children Questionnaire (HBSC). The HBSC was also administered to assess the influence of stress on eating behavior: the consumption of fruit, vegetables, sweets, and soft drinks and the frequency of snacks [16]

\subsection{Collection and Analysis of Salivary Cortisol Samples}

Saliva samples were obtained $2 \mathrm{~min}$ before the experiment, $2 \mathrm{~min}$ after each digital test, and also at the end of the test (T0, T5, T10, T15, and T40) using the same technique. Briefly, a neutral cotton-based swab, SalivetteTM (Sarstedt, Numrecht, Germany), was chewed for about $2 \mathrm{~min}$ and then placed in a double-lumen tube. The tube was submitted to the laboratory and refrigerated until it was centrifuged at $1000 \mathrm{rpm}$ for $5 \mathrm{~min}$ at room temperature. After this, the saliva was frozen to $-20^{\circ} \mathrm{C}$ before analysis. A commercial direct immunoenzymatic method for salivary cortisol, DKO020 Cortisol Saliva ELISA (Diametra, Italy), was used, with $0.12 \mathrm{ng} / \mathrm{mL}$ sensitivity and an analytical range of $0.5-100$.

Additionally, the area under the curve (AUC) with respect to ground $\left(\mathrm{AUC}_{\mathrm{g}}\right)$ was calculated [18].

\subsection{Anthropometric Measurements}

Assessments were performed by the same group of extensively experienced researchers. Height and weight were measured for each participant. These measurements were taken with the students in light clothes and without shoes. Body mass was measured on a digital scale (Healthy Line, SHL-9015B, CE) with an accuracy of $\pm 100 \mathrm{~g}$. The height of the children was measured with a portable stadiometer (KaWe, Germany) with an accuracy of $\pm 0.1 \mathrm{~cm}$.

Actual BMI $\left(\mathrm{kg} / \mathrm{m}^{2}\right)$ was calculated by dividing weight $(\mathrm{kg})$ by height squared $(\mathrm{m})$. BMI was classified based on World Health Organization (WHO) guidelines [15].

\subsection{Statistical Analysis}

Data were analyzed using the Statistical Package for the Social Sciences (SPSS) version 22 (IBM Corp., Armonk, NY, USA). Continuous variables were presented as mean (SD) or median, as appropriate. Statistical calculations were performed using a non-parametric test since the salivary cortisol data were classified as not normally distributed by the Shapiro-Wilk normality test. The comparison of two independent values was analyzed by the Mann-Whitney test. Pearson's correlation was used to investigate the relationship between food consumption and the subjects' stress response. The effect of the TSST on salivary cortisol was examined by analysis of variance (ANOVA) with repeated measures. Differences were considered significant at $p<0.05$.

\section{Results}

3.1. Sociodemographics, Anthropometric, Perceived Stress, and Cortisol Response to Induced-Social Stress in Adolescents

Participants' sociodemographic characteristics, BMIs, perceived stress scores, and salivary cortisol are displayed in Table 1 . There were no significant differences in the sociodemographic, anthropometric, physical activity, general health and well-being, and perceived stress variables between boys and girls. 
Table 1. The characteristics of studied adolescents.

\begin{tabular}{|c|c|c|c|}
\hline Characteristics & $\begin{array}{c}\text { Boys } \\
(N=16)\end{array}$ & $\begin{array}{c}\text { Girls } \\
(N=19)\end{array}$ & $p$-Value \\
\hline \multicolumn{4}{|l|}{ Sociodemographic parameters } \\
\hline Age & $15.6 \pm 0.7$ & $15.5 \pm 0.6$ & 0.739 \\
\hline Mother's education (high scool or less, \%) & 50 & 57.1 & 0.606 \\
\hline Father's education (high scool or less, \%) & 33.4 & 42.9 & 0.732 \\
\hline $\begin{array}{l}\text { How many people live in your house? } \\
(\geq 4, \%)\end{array}$ & 50 & 57.2 & 0.878 \\
\hline $\begin{array}{l}\text { Perceived family wealth (very well off and } \\
\text { well off, \%) }\end{array}$ & 87.5 & 94.7 & 0.453 \\
\hline \multicolumn{4}{|l|}{ Anthropometric parameters } \\
\hline $\operatorname{BMI}\left(\mathrm{kg} / \mathrm{m}^{2}\right)$ & $21.8 \pm 3.6$ & $21.52 \pm 2.7$ & 0.855 \\
\hline Excess weight ${ }^{1}(\% / N)$ & $43.8(7)$ & $21.1(4)$ & 0.286 \\
\hline \multicolumn{4}{|l|}{ Physical activity } \\
\hline Vigorous exercise $1 \mathrm{~h}$ ( $\geq 3$ days/week, \%) & 37.5 & 47.4 & 0.562 \\
\hline $\begin{array}{l}\text { Playing computer games }(\geq 2 \mathrm{~h} / \text { school } \\
\text { day, \%) }\end{array}$ & 14.3 & 58.3 & 0.106 \\
\hline Watching TV ( $\geq 2$ h/school day, \%) & 60 & 68.4 & 0.071 \\
\hline \multicolumn{4}{|l|}{ General health and well-being } \\
\hline Self-rated health (excellent and good, \%) & 50 & 26,3 & 0.155 \\
\hline $\begin{array}{l}\text { Multiple complaints }(\geq 2 \\
\text { complaints/week, } \%)\end{array}$ & 100 & 94.7 & 0.359 \\
\hline \multicolumn{4}{|l|}{ Perceived stress } \\
\hline Pre-test score (mean \pm SD, median) & $21.09 \pm 7.37(23)$ & $24,81 \pm 6,19$ & 0.167 \\
\hline Post-test score (mean $\pm \mathrm{SD}$, median) & $29.97 \pm 7.34(31)$ & $31,76 \pm 5,49$ & 0.231 \\
\hline \multicolumn{4}{|l|}{ Cortisol reactivity to TSST } \\
\hline \multicolumn{4}{|l|}{ Salivary cortisol (nmol/L) } \\
\hline T0 (before experiment) & $4.3 \pm 2.9$ & $5.8 \pm 3.3$ & 0.145 \\
\hline T5 (after the 1st digital test) & $10.4 \pm 10.6$ & $10.5 \pm 9.3$ & 0.655 \\
\hline T10 (after the 2nd digital test) & $27.1 \pm 28.0$ & $26.1 \pm 23.7$ & 0.947 \\
\hline T15 (after the 3rd digital test) & $25.1 \pm 27.5$ & $36.3 \pm 31.4$ & 0.246 \\
\hline T40 (after 25 min of recovery) & $12.6 \pm 9.7$ & $25.5 \pm 20.1$ & $0.020 *$ \\
\hline Area under the curve, AUGg & $690.1 \pm 658.2$ & $1087.8 \pm 813.6$ & 0.091 \\
\hline
\end{tabular}

${ }^{1}$ According to the age and specific cutoff points for BMI, as published by the WHO [15]. The data are presented as the mean \pm standard deviation (SD) or median Mann-Whitney U test used to compare variables. TSST, Trier social stress test. * $p$-value significantly different between groups $(p<0.05)$.

The TSST induced significant effects of time interaction for cortisol $(\mathrm{F}(4,31)=11,48$, $p<0.001$ ). The difference in cortisol levels between boys and girls (using the MannWhitney U test) was significant at the T40 time point $(p=0.020)$.

\subsection{Cortisol Reactivity and Adiposity in Adolescents}

Depending on the cortisol response to the digital version of the TSST, participants were categorized into hypo (AUCg $<501.5 \mathrm{nmol} / \mathrm{L} * \min , \mathrm{n}=17$ ) and hyper-reactive (AUCg $>501.5 \mathrm{nmol} / \mathrm{L} *$ min, $\mathrm{n}=18$ ) groups according to the median value of AUCg (Figure 1). Among the entire group of adolescents, we observed a significant difference between the sexes in terms of cortisol response, girls being predominantly hyperreactive (13 girls compared to 5 boys, $p=0.03$ ).The frequency of non-overweight adolescents with higher reactivity of cortisol was nonsignificantly higher in girls as compared to boys $(p=0.295)$. In overweight adolescents, the cortisol reactivity was lower in boys and higher in girls $(p=0.05$ (Figure 1$)$ ). 


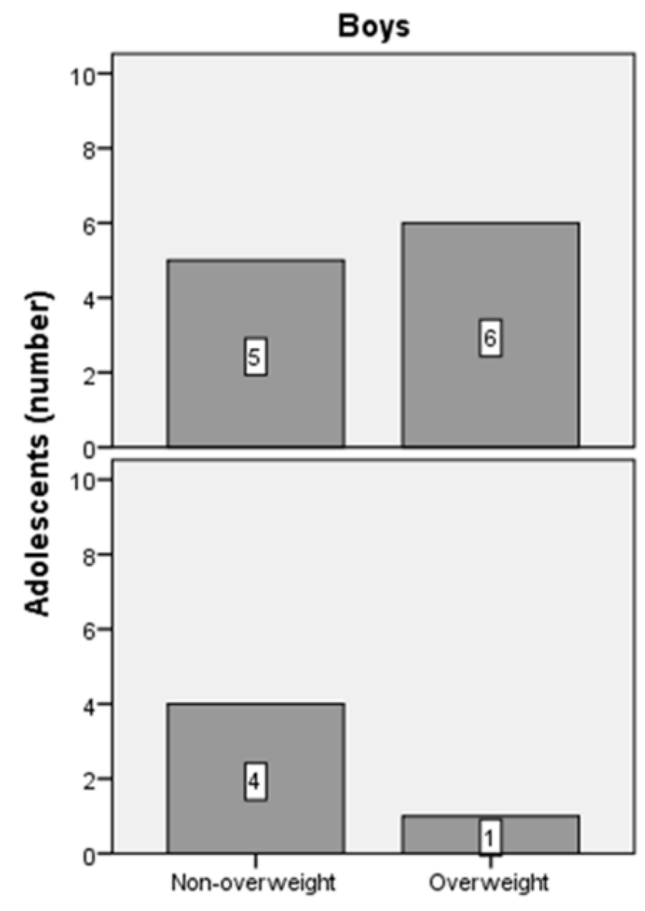

(a)

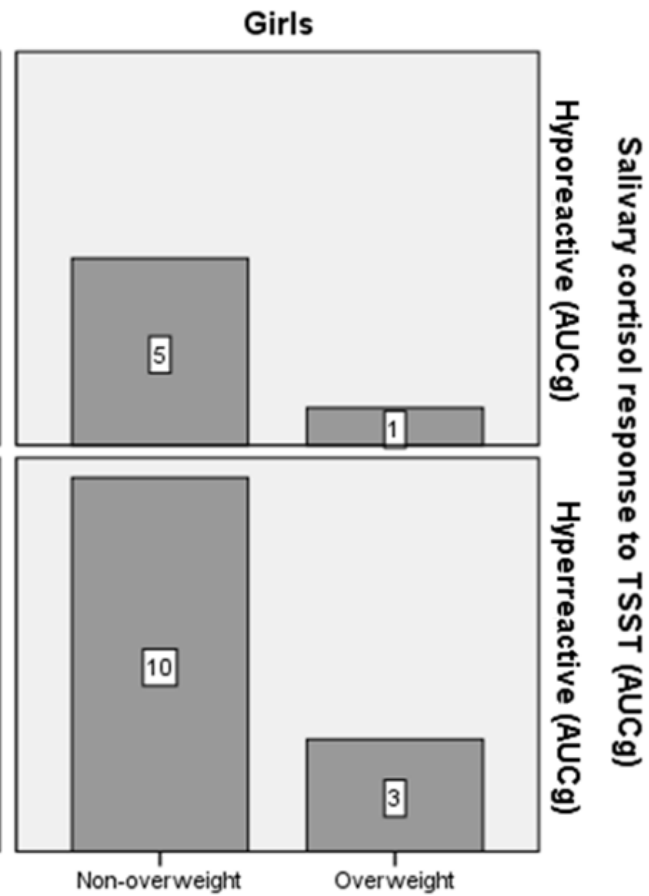

(b)

Figure 1. The frequency of non-overweight and overweight adolescents with hypo or hyper-reactivity of salivary cortisol response to TSST. (a) Boys; (b) girls.

\subsection{Cortisol Reactivity and Eating Behavior in Adolescents}

Among the entire group of adolescents, we found non-significant differences between hypo and hyperreactive adolescents regarding the daily consumption of snacks, fruit, vegetables, and sweets (Table 2). The adolescents from the hyperreactive group had significantly increased consumption of soft drinks (sweetened beverages) as compared to the hyporeactive group $(p=0.021)$. The non-overweight hyperreactive adolescents, as compared to the hyporeactive ones, had a higher frequency of snack consumption, $>3$ daily ( $29 \%$ vs. $10 \%)$, consumption of sweets ( $14 \%$ vs. $10 \%)$, and soft drink consumption ( $29 \%$ vs. 0 ). The overweight hyperreactive adolescents, as compared to the hyporeactive ones, had a higher frequency of daily consumption of sweets ( $25 \%$ vs. $0 \%)$ and soft drink ( $25 \%$ vs. 0$)$.

Table 2. The food behavior in adolescents and their salivary cortisol response (low or high) to TSST.

\begin{tabular}{cccc}
\hline Food Consumption & $\begin{array}{c}\text { Hyporeactive } \\
(\mathbf{N}=\mathbf{1 7})\end{array}$ & $\begin{array}{c}\text { Hyperreactive } \\
(\mathbf{N}=\mathbf{1 8})\end{array}$ & $p$-Value \\
\hline Snack frequency (>3 daily) (\%) & 5.9 & 22.2 & 0.174 \\
Snack frequency (>6 daily) (\%) & 11.8 & 5.6 & 0.303 \\
Fruit consumption (daily) (\%) & 35.3 & 16.7 & 0.214 \\
Vegetable consumption (daily) (\%) & 29.4 & 44.4 & 0.365 \\
Consumption of sweets (daily) (\%) & 5.9 & 16.7 & 0.323 \\
Soft drink consumption (daily) (\%) & 0 & 27.8 & $0.021{ }^{*}$ \\
\hline
\end{tabular}

$p$-value was obtained using the Mann-Whitney $\mathrm{U}$ test. ${ }^{*} p$-value $\leq 0.05$ was statistically significant.

The hyper-reactive boys, as compared to the hyporeactive ones, had a higher frequency vegetable consumption ( $40 \%$ vs. $9 \%$ ) and soft drink consumption ( $40 \%$ vs. 0 ). The hyperreactive girls, as compared to the hyporeactive ones, had a higher frequency of snack consumption, $>3$ daily ( $31 \%$ vs. $17 \%)$, consumption of sweets ( $23 \%$ vs. $17 \%)$, and soft drink consumption ( $23 \%$ vs. 0). (Figure 2$)$. 


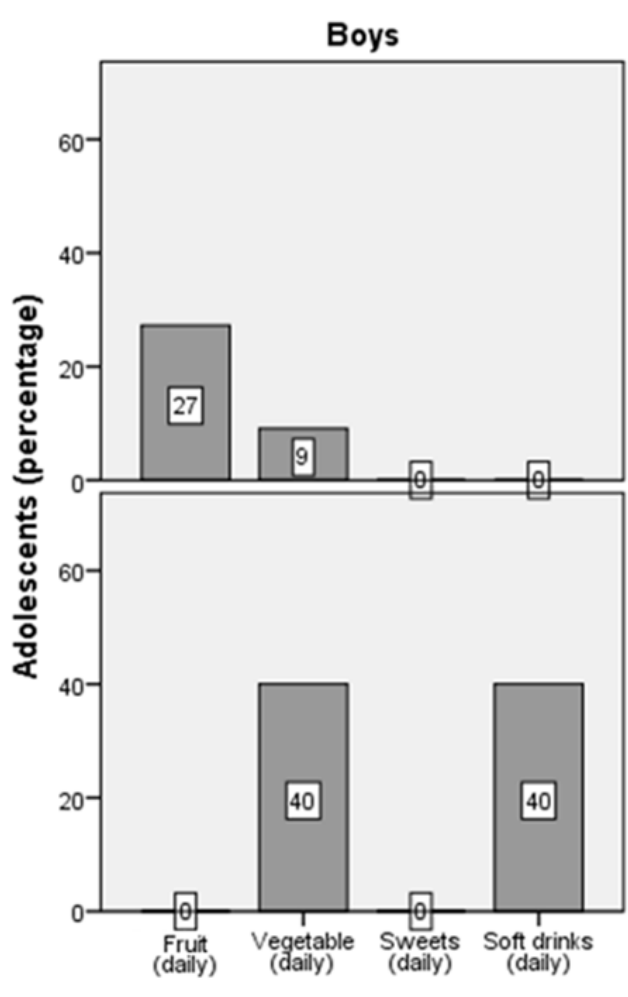

(a)

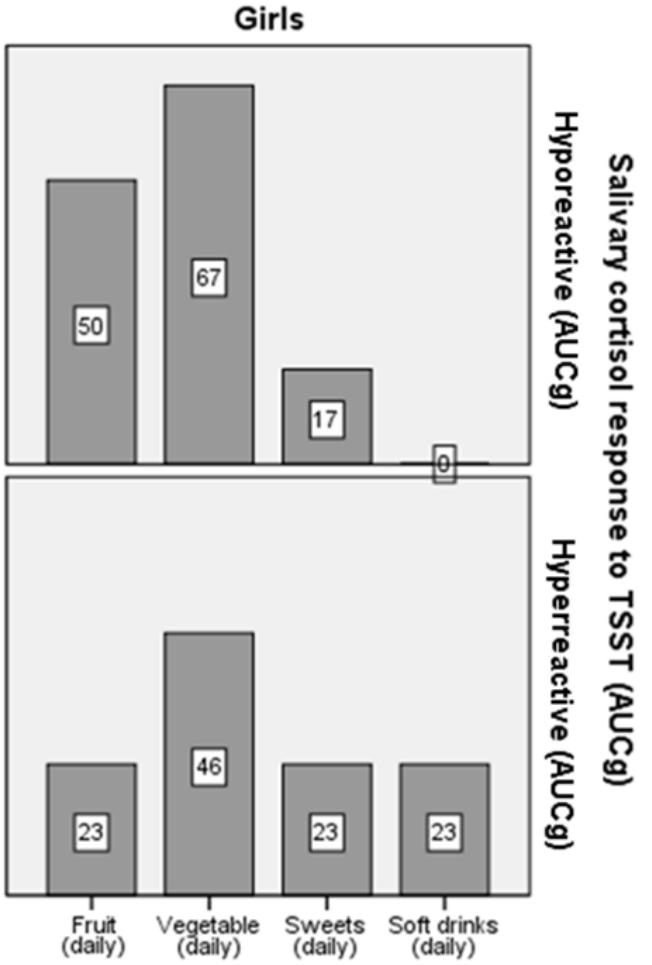

(b)

Figure 2. The food consumption in adolescents with hypo or hyper-reactivity of salivary cortisol to TSST. (a) Boys; (b) girls.

\subsection{Correlations between Cortisol Response to TSST and PSS Scores}

There were significant positive correlations between AUCg and the PSS pre-test $(\mathrm{r}=0.345, p=0.043)$ and post-test $(\mathrm{r}=0.483, p=0.003)$ scores.

\subsection{Correlations between Stress Parameters and Eating Behavior}

We found a tendency to a significant positive correlation between AUCg and soft drink consumption $(r=0.303, p=0.077)$ in all groups of adolescents. In overweight adolescents $(\mathrm{N}=11)$, we found significant positive correlations between AUCg and consumption of sweets $(r=0.698, p=0.017)$ and soft drinks $(r=0.813, p=0.002)$. The PSS posttest score postively correlated with the consumption of sweets $(r=0.621, p=0.042)$ in overweight adolescents.

\section{Discussion}

In this pilot study, we examined the cortisol response to a digital version of the induced acute psychological stress test (Trier Social Stress Test, TSST) in a group of adolescents. We also assessed the eating behavior (using the Health Behavior in School-Aged Children, HBSC, Questionnaire) and its relationship with the characteristics of cortisol reactivity during the TSST. Our results indicate that eating habits are related to the hypo or hyperreactivy of salivary cortisol response to the TSST. Hyperreactive adolescents ate fewer fruits and drank more soft drinks. Three of four overweight girls had hyperreactive cortisol response, and six of seven overweight boys had hyporeactive cortisol response. The characteristics of eating behavior in overweight adolescents suggested by our study are: (1) overweight boys were hyporeactive to induced psychological stress and had an increased frequency of snacks as compared to the normal-weight group; (2) overweight girls were hyperreactive to induced psychological stress and had an increased consumption of soft drinks. In overweight adolescents, positive correlations were noticed between salivary cortisol response (AUCg) and unhealthy food consumption (sweets and soft drinks). Additionally, 
the perceived stress score (Perceived Stress Scale) post-TSST positively correlated with consumption of sweets.

Previous studies have demonstrated an increased ad libitum food intake in healthy individuals with a high cortisol reactivity in response to a laboratory stressor $[2,19,20]$. In people with obesity, diverse outcomes have been reported on the link between stressinduced cortisol reactivity and food behavior. Higher cortisol reactivity in response to the standard TSST was associated with increased food consumption $[2,5,20]$ or reduced snack intake [21] in obese women with high cortisol reactivity.

Moss et al. [14] studied children's eating behavior for 7 consecutive days, asking subjects to complete a diary before going to bed. Each daily diary asked about: the day's events, snacks consumed, and their feelings. In this study, the consumption of unhealthy snacks was strongly associated with daily negative moods in children with higher levels of AUCg.

The cortisol reactivity to the TSST tends to be significantly higher in non-obese men as compared to non-obese women, but no differences were observed between men and women with obesity [1]. In women with obesity and binge eating disorder (BED) the cortisol reactivity to the TSST was lower as compared to normal-weight and obese women [6]. The blunted pattern of cortisol response to an acute stressor was explained by a possible compensatory down-regulation of the HPA axis secondary to the presence of chronic stress in this group of obese BED participants.

The importance of the current study is to demonstrate that the relationship between the cortisol reactivity to a particular digital version of the TSST (AUCg) and eating behavior has different patterns in non-overweight and overweight adolescents. In the studied adolescents, the percentage of overweight boys was very high (43.8\%). The overweight boys were hyporeactive, with an increased frequency of snack and soft drink consumption. This could suggest a down-regulation of the HPA axis secondary to chronic stress [7,22,23] and the increase in comfort food intake as a compensatory mechanism [6]. The lower cortisol response to the TSST could also suggest increased abdominal adiposity [24] or lower perceived stress [24]. The overweight girls (21.1\% of total) were hyperreactive (three of four), suggesting increased activation of the HPA axis following an acute stress. In girls, higher cortisol reactivity was positively associated with depressed mood and higher BMI [12]. Pubertal girls respond to stress by turning to high-calorie foods, and this behavior may then be reinforced by physiological reward pathways [25]. Moreover, significant correlations between cortisol responses and perceived stress scores were found using our digital version of the TSST. By comparison, approximately $25 \%$ of the studies using the standard TSST have found correspondence between physiological arousal and subjective emotional stress experience [26].

Strengths and limitations. This is the first study using tablet display technology to evaluate cortisol response to psychosocial stress and its impact on eating behavior. The proposed digital version of the Trier Social Stress Test (TSST) is a standardized, inexpensive, and portable method. The group of adolescents who participated in the experiment was quite homogeneous (high school students), and all participants carried out the stress test under the same external conditions, in a classroom of their school. A possible limitation of our study is the modest number of participants $(\mathrm{N}=35$, with only 17 adolescents with hyporeactivity and 18 adolescents with hyperreactivity to acute psychologic stress). Another limitation is the lack of a control group against which to compare the variables studied. The study needs to be replicated on a larger scale to validate the initial results.

\section{Conclusions}

The individual patterns of cortisol reactivity to laboratory-induced stress could be associated with an increased risk of unhealthy eating behavior in adolescents. The digital version of the TSST proposed in this study is a reliable, easy-to-use, and suitable method for assessing HPA dysfunctionalities and could be beneficial in the diagnosis and management of overweight adolescents. 
Author Contributions: Conceptualization, I.G. and V.M.; methodology, I.G., V.M., C.M.G. and D.V.T.; investigation, I.G., D.A, D.-T.A.-P. and L.L.; data curation, I.G., A.D., C.M.G., M.C.U. and C.P.; writing—original draft preparation, I.G. and A.D.; writing—review and editing, V.M. All authors have read and agreed to the published version of the manuscript.

Funding: This research received no external funding.

Institutional Review Board Statement: The study was conducted according to the guidelines of the Declaration of Helsinki and approved by the Research Ethics Committee of the University of Medicine and Pharmacy from Iasi (15304/22 July 2019).

Informed Consent Statement: An informed consent form was signed by the school directors, parents, and students.

Acknowledgments: We want to thank the school principals, classroom teachers, and all of the students for participating in this study.

Conflicts of Interest: The authors declare no conflict of interest.

\section{References}

1. Therrien, F.; Drapeau, V.; Lalonde, J.; Lupien, S.J.; Beaulieu, S.; Tremblay, A.; Richard, D. Awakening cortisol response in lean, obese, and reduced obese individuals: Effect of gender and fat distribution. Obesity 2007, 15, 377-385. [CrossRef]

2. Epel, E.; Lapidus, R.; McEwen, B.; Brownell, K. Stress may add bite to appetite in women: A laboratory study of stress-induced cortisol and eating behavior. Psychoneuroendocrinology 2001, 26, 37-49. [CrossRef]

3. Dickerson, S.S.; Kemeny, M.E. Acute stressors and cortisol responses: A theoretical integration and synthesis of laboratory research. Psychol. Bull. 2004, 130, 355-391. [CrossRef]

4. Gluck, M.E.; Geliebter, A.; Hung, J.; Yahav, E. Cortisol, hunger, and desire to binge eat following a cold stress test in obese women with binge eating disorder. Psychosom. Med. 2004, 66, 876-881. [CrossRef] [PubMed]

5. Herhaus, B.; Ullmann, E.; Chrousos, G.; Petrowski, K. High/low cortisol reactivity and food intake in people with obesity and healthy weight. Transl. Psychiatry 2020, 10, 40. [CrossRef]

6. Rosenberg, N.; Bloch, M.; Ben Avi, I.; Rouach, V.; Schreiber, S.; Stern, N.; Greenman, Y. Cortisol response and desire to binge following psychological stress: Comparison between obese subjects with and without binge eating disorder. Psychiatry Res. 2013, 208, 156-161. [CrossRef] [PubMed]

7. Kirschbaum, C.; Pirke, K.M.; Hellhammer, D.H. The 'Trier Social Stress Test' - A tool for investigating psychobiological stress responses in a laboratory setting. Neuropsychobiology 1993, 28, 76-81. [CrossRef]

8. Schulz, S.; Laessle, R.G. Stress-induced laboratory eating behavior in obese women with binge eating disorder. Appetite 2012, 58, 457-461. [CrossRef]

9. Herhaus, B.; Passler, S.; Petrowski, K. Stress-related laboratory eating behavior in adults with obesity and healthy weight. Physiol. Behav. 2018, 196, 150-157. [CrossRef] [PubMed]

10. Liang, J.; Matheson, B.E.; Kaye, W.H.; Boutelle, K.N. Neurocognitive correlates of obesity and obesity-related behaviors in children and adolescents. Int. J. Obes. 2014, 38, 494-506. [CrossRef]

11. Verdejo-Garcia, A.; Moreno-Padilla, M.; Garcia-Rios, M.C.; Lopez-Torrecillas, F.; Delgado-Rico, E.; Schmidt-Rio-Valle, J.; Fernandez-Serrano, M.J. Social stress increases cortisol and hampers attention in adolescents with excess weight. PLoS ONE 2015, 10, e0123565. [CrossRef]

12. Lu, Q.; Tao, F.; Hou, F.; Zhang, Z.; Sun, Y.; Xu, Y.; Xu, S.; Zhao, Y. Cortisol reactivity, delay discounting and percent body fat in Chinese urban young adolescents. Appetite 2014, 72, 13-20. [CrossRef]

13. Messerli-Burgy, N.; Horsch, A.; Schindler, C.; Boichat, A.; Kriemler, S.; Munsch, S.; Crottet, B.; Marquez-Vidal, P.M.; Borghini, A.; Puder, J.J. Influence of Acute Physical Activity on Stress Reactivity in Obese and Normal Weight Children: A Randomized Controlled Trial. Obes. Facts 2019, 12, 115-130. [CrossRef]

14. Moss, R.H.; Conner, M.; O'Connor, D.B. Exploring the effects of daily hassles on eating behaviour in children: The role of cortisol reactivity. Psychoneuroendocrinology 2020, 117, 104692. [CrossRef]

15. World Health Organization. Growth Reference 5-19 Years-BMI-for-Age (5-19 Years). 2007. Available online: https://www.who. int/tools / growth-reference-data-for-5to19-years/indicators/bmi-for-age (accessed on 22 August 2021).

16. Currie, C.; Nic Gabhainn, S.; Godeau, E.; International, H.N.C.C. The Health Behaviour in School-aged Children: WHO Collaborative Cross-National (HBSC) study: Origins, concept, history and development 1982-2008. Int. J. Public Health 2009, 54 (Suppl. 2), 131-139. [CrossRef]

17. Cohen, S.; Kamarck, T.; Mermelstein, R. A global measure of perceived stress. J. Health Soc. Behav. 1983, 24, 385-396. [CrossRef]

18. Khoury, J.E.; Gonzalez, A.; Levitan, R.D.; Pruessner, J.C.; Chopra, K.; Basile, V.S.; Masellis, M.; Goodwill, A.; Atkinson, L. Summary cortisol reactivity indicators: Interrelations and meaning. Neurobiol. Stress 2015, 2, 34-43. [CrossRef] [PubMed]

19. George, S.A.; Khan, S.; Briggs, H.; Abelson, J.L. CRH-stimulated cortisol release and food intake in healthy, non-obese adults. Psychoneuroendocrinology 2010, 35, 607-612. [CrossRef] 
20. Newman, E.; O'Connor, D.B.; Conner, M. Daily hassles and eating behaviour: The role of cortisol reactivity status. Psychoneuroendocrinology 2007, 32, 125-132. [CrossRef] [PubMed]

21. Appelhans, B.M.; Pagoto, S.L.; Peters, E.N.; Spring, B.J. HPA axis response to stress predicts short-term snack intake in obese women. Appetite 2010, 54, 217-220. [CrossRef] [PubMed]

22. Cunningham, S.; Mazurka, R.; Wynne-Edwards, K.E.; Milev, R.V.; Pizzagalli, D.A.; Kennedy, S.; Harkness, K.L. Cortisol reactivity to stress predicts behavioral responsivity to reward moderation by sex, depression, and anhedonia. J. Affect. Disord. 2021, 293, 1-8. [CrossRef] [PubMed]

23. Mazurka, R.; Wynne-Edwards, K.E.; Harkness, K.L. Stressful Life Events Prior to Depression Onset and the Cortisol Response to Stress in Youth with First Onset Versus Recurrent Depression. J. Abnorm. Child Psychol. 2016, 44, 1173-1184. [CrossRef] [PubMed]

24. Krishnaveni, G.V.; Jones, A.; Veena, S.R.; Somashekara, R.; Karat, S.C.; CH, D.F. Adiposity and Cortisol Response to Stress in Indian Adolescents. Indian Pediatr. 2018, 55, 125-130. [CrossRef] [PubMed]

25. Dockray, S.; Susman, E.J.; Dorn, L.D. Depression, cortisol reactivity, and obesity in childhood and adolescence. J. Adolesc. Health 2009, 45, 344-350. [CrossRef]

26. Campbell, J.; Ehlert, U. Acute psychosocial stress: Does the emotional stress response correspond with physiological responses? Psychoneuroendocrinology 2012, 37, 1111-1134. [CrossRef] 